\title{
Mobility Analysis of Generalized Angulated Scissor-like Elements with the Reciprocal Screw Theory
}

\author{
Jianguo Cai ${ }^{1,{ }^{*}}$, Xiaowei Deng ${ }^{2}$, Jian Feng ${ }^{1}$, Yixiang $\mathrm{Xu}^{3}$ \\ ${ }^{1}$ Key Laboratory of C \& PC Structures of Ministry of Education, National Prestress Engineering \\ Research Center, Southeast University, Nanjing 210096, China \\ ${ }^{2}$ Department of Structural Engineering, University of California, San Diego, USA \\ ${ }^{3}$ Department of Civil Engineering, Strathclyde University, Glasgow G12 8QQ, United Kingdom
}

Abstract: This paper deals with the planar closed loop linkage consisting of a series of scissor-like elements connected by revolute joints. Because every generalized angulated element (GAE) subtends a constant angle during the motion, every angulated link was assumed as a PRRP linkage which has two prismatic joints and two revolute joints. Therefore, the two PRRP linkages of the GAE are individually movable with a single degree of freedom. The mobility of two types of GAEs was investigated with the method based on the screw theory. It has been proven that both types of GAEs are movable because the terminal constraints exerted to the common joint by the two linkages are equal.

Keywords: kinematics; scissor-like element; linkages; screw theory

\footnotetext{
"corresponding author. Fax: 0086-025-8379-5150.

Email: caijg_ren@hotmail.com, j.cai@seu.edu.cn (Cai Jianguo)
} 


\section{Introduction}

Foldable structures, which can be transformed from a closed configuration to a expanded form, are developed from the earliest modern stages with Pinero's movable theater in 1961 [1]. Especially during the last decade, the retractable roof structures are widely used in long-span space structures [2]. On the other hand, with the further development of aerospace science and technology, deployable structures are of even greater interest in aerospace industries $[3,4]$.

Generally, the deployable structure based on the scissor-like element is the most commonly used form in engineering [5-8]. A scissor-like element in two dimensional forms is shown in Fig.1 (a). The axis of the revolute joint is perpendicular to the plane of the structure. The element has one degree-of-freedom and can be folded and deployed freely. Besides the foldable truss and dome structures [9], Zhao et al. $[10,11]$ proposed a foldable stair, which consists of a number of identical deployable scissor-like elements. In the early 1990's, Hoberman [12, 13] invented and patented a method for constructing loop assemblies formed by the modified scissor-like element, which consists of a pair of identical angulated rods connected by a revolute joint as shown in Fig.1 (b). Therefore, the unit is also called angulated scissor element or Hoberman's unit. You and Pellegrino [14] noted that the unit subtends a constant angle as their rods rotate while maintaining the end pivots on parallel lines. Thus the Hoberman's unit can create a closed-loop mechanism, which is called Hoberman's Linkage as shown in Fig.2. They also proposed two types of generalized angulated elements (GAEs), which subtend a constant angle during folding, but afford much 
greater freedom of shapes than Hoberman's unit. The triangles of the two angulated rods of Type I GAE are isosceles triangles, i.e. $\mathrm{AE}=\mathrm{DE}$ and $\mathrm{BE}=\mathrm{CE}$, as shown in Fig.3. The triangles of the two angulated rods of Type II GAE are similar triangles, i.e.

$\mathrm{AE} / \mathrm{BE}=\mathrm{DE} / \mathrm{CE}$ and $\angle \mathrm{AEB}=\angle \mathrm{DEC}$. As shown in Fig.4. the lengths of $\mathrm{BE}$ and $\mathrm{AE}$ are $p$ and $q$. For Type II GAE, $\mathrm{AE} / \mathrm{BE}=\mathrm{DE} / \mathrm{CE}=w$. Then the lengths of $\mathrm{CE}$ and $\mathrm{DE}$ are $w p$ and $w q$. The planar closed loop linkages which include Type I GAEs and Type II GAEs are given in Fig.5.

The focus of this paper is on the mobility analysis of this planar closed loop linkage. It is pertinent to point out that the kinematic treatment of linkages is receiving increasing attention in recent years [15-18]. Langbecker [5] proposed the foldability equation of deployable scissor structures, which is formulated using a purely geometric approach. Patel and Ananthasuresh [16] presented a kinematic theory behind Hoberman's and other inventions related to planar, radially foldable linkages. They showed that known types of foldable linkages can be derived by using a simple algebraic equation and even simpler design criteria. Based on the corresponding mobility conditions, Mao et al. [17] produced mobile double chains with both even and odd number of intersecting scissor-like pairs. A numerical algorithm was used by Nagaraj et al. [18] to evaluate the degree-of-freedom of pantograph masts by obtaining the null space of a constraint Jacobian matrix. Moreover, the principles of screw theory can also be used to study the degree-of-freedom of mechanism. Dai et al. [19-21] studied the mobility of a general type of foldable mechanisms using the screw theory. Zhao et al. [22] applied the screw theory to study the degree-of-freedom of simple 
planar linkage and the mechanism theory of forming the spatial deployable units utilized in flat, cylindrical and spherical deployable structures. But they only focused on the scissor-like elements with straight rods. Then Cai et al. [23] extend this theory to the mobility analysis of the Hoberman's angulated unit. Dai et al. [24] and Wei et al. [25, 26] also used the reciprocal screws to study the mobility of the Hoberman structure and its variant. However, the scissor-like elements studied in the previous literatures are very simple and the geometry of these elements is symmetry. Two types of GAEs proposed by You and Pellegrino [14], which are not considered in these literatures, have asymmetry geometry and complex kinematic behavior. The mobility of GAEs will be studied in this paper with the screw theory. Prior to probing the mechanism theory of the GAE in Section 3, the kinematic description of GAEs will be given in Section 2. Section 4 draws conclusions.

\section{Kinematic description of GAEs}

As mentioned earlier, the main attribute of the planar closed loop linkages studied in this paper is that every angulated element subtends a constant angle during the motion. A simple generalized angulated element is shown in Fig.6a. The common revolute joint connects the two angulated rods of equal configuration. Therefore, points A, B, C and D can be seen as to be constrained to move along either of the dash lines $\mathrm{P}_{1}$ and $\mathrm{P}_{2}$. Then we can interpret an angulated link as a PRRP linkage shown in Fig. 6(b), which has two prismatic joints and two revolute joints. Thus the two PRRP linkages of the GAE are individually movable with a single degree of freedom. Therefore, the GAE will be 
movable only when the two linkages share the same coupler curve at point E [16].

\section{Kinematic analysis of GAEs}

The GAE can be considered as a parallel mechanism. It consists of the linkage ABE and linkage CDE, which are connected by a common joint E. Its mobility can be analyzed by the method based on the screw theory, as proposed in Ref. [24].

It is assumed that the subtended angle between line $\mathrm{AD}$ and line $\mathrm{BC}$ is denoted by $2 \alpha$. The orthogonal coordinate oxy is shown in Fig. 7. The axis $o x$ is the bisector of angle $\angle \mathrm{BOD}$, and the axis $o y$ is perpendicular to the $o x$ axis in the mechanism plane. Now, the kinematics of the link ABE is studied firstly.

There are a revolute joint and a slide joint at point A. Then the twists of the RP chain are given as

$$
\boldsymbol{\Phi}_{A}=\left[\begin{array}{ll}
\boldsymbol{\Phi}_{A_{R}} & \boldsymbol{\Phi}_{A_{S}}
\end{array}\right]
$$

where

$$
\begin{gathered}
\boldsymbol{\Phi}_{A_{R}}=\left\{\begin{array}{lllllll}
0 & 0 & 1 & y_{A} & -x_{A} & 0
\end{array}\right\}^{T} \\
\boldsymbol{\Phi}_{A_{S}}=\left\{\begin{array}{llllll}
0 & 0 & 0 & \cos \alpha & -\sin \alpha & 0
\end{array}\right\}^{T}
\end{gathered}
$$

and $x_{\mathrm{A}}, y_{\mathrm{A}}$ are the coordinates of point $\mathrm{A}$.

According to the screw theory, the terminal constraint screw matrices of the RP chain are immediately obtained by solving the reciprocal screw equations

$$
\boldsymbol{\Phi}_{A}{ }^{T} \mathbf{E} \boldsymbol{\Gamma}_{A}=0
$$

where $\mathbf{E}$ is the swap operator and its formulation is expressed as follows 


$$
\mathbf{E}=\left[\begin{array}{cc}
0 & \mathbf{I}_{3} \\
\mathbf{I}_{3} & 0
\end{array}\right] \text { and } \quad \mathbf{I}_{3}=\left[\begin{array}{lll}
1 & 0 & 0 \\
0 & 1 & 0 \\
0 & 0 & 1
\end{array}\right]
$$

Therefore, the terminal constraints external to link ABE by the RP chain are

$$
\boldsymbol{\Gamma}_{A}=\left[\begin{array}{llll}
\boldsymbol{\Gamma}_{A, 1} & \boldsymbol{\Gamma}_{A, 2} & \boldsymbol{\Gamma}_{A, 3} & \boldsymbol{\Gamma}_{A, 4}
\end{array}\right]\left[\begin{array}{l}
f_{1} \\
f_{2} \\
f_{3} \\
f_{4}
\end{array}\right]
$$

where $f_{\mathrm{i}}(i=1,2,3,4)$ represent the tensities of the screws and

$$
\begin{aligned}
& \boldsymbol{\Gamma}_{A, 1}=\left\{\begin{array}{llllll}
-\sin \alpha & -\cos \alpha & 0 & 0 & 0 & \left.-x_{A} \cos \alpha+y_{A} \sin \alpha\right\}^{T}
\end{array}\right. \\
& \boldsymbol{\Gamma}_{A, 2}=\left\{\begin{array}{llllll}
0 & 0 & 1 & 0 & 0 & 0
\end{array}\right\}^{T} \\
& \boldsymbol{\Gamma}_{A, 3}=\left\{\begin{array}{llllll}
0 & 0 & 0 & 1 & 0 & 0
\end{array}\right\}^{T} \\
& \boldsymbol{\Gamma}_{A, 4}=\left\{\begin{array}{llllll}
0 & 0 & 0 & 0 & 1 & 0
\end{array}\right\}^{T}
\end{aligned}
$$

Similarly, the terminal constraints external to link ABE by the RP chain at point B are

$$
\boldsymbol{\Gamma}_{B}=\left[\begin{array}{llll}
\boldsymbol{\Gamma}_{B, 1} & \boldsymbol{\Gamma}_{B, 2} & \boldsymbol{\Gamma}_{B, 3} & \boldsymbol{\Gamma}_{B, 4}
\end{array}\right]\left[\begin{array}{l}
f_{5} \\
f_{6} \\
f_{7} \\
f_{8}
\end{array}\right]
$$

where

$$
\begin{aligned}
& \boldsymbol{\Gamma}_{B, 1}=\left\{\begin{array}{llllll}
\sin \alpha & -\cos \alpha & 0 & 0 & 0 & \left.-x_{B} \cos \alpha-y_{B} \sin \alpha\right\}^{T}
\end{array}\right. \\
& \boldsymbol{\Gamma}_{B, 2}=\left\{\begin{array}{llllll}
0 & 0 & 1 & 0 & 0 & 0
\end{array}\right\}^{T} \\
& \boldsymbol{\Gamma}_{B, 3}=\left\{\begin{array}{llllll}
0 & 0 & 0 & 1 & 0 & 0
\end{array}\right\}^{T} \\
& \boldsymbol{\Gamma}_{B, 4}=\left\{\begin{array}{llllll}
0 & 0 & 0 & 0 & 1 & 0
\end{array}\right\}^{T}
\end{aligned}
$$

$f_{\mathrm{i}}(i=5,6,7,8)$ represent the tensities of the screws and $x_{\mathrm{B}}, y_{\mathrm{B}}$ are the coordinates of point B.

We can find the terminal constraints exerted to link ABE using Eqs. (3) and (4) as 


$$
\boldsymbol{\Gamma}_{A B E}=\left[\begin{array}{lllll}
\boldsymbol{\Gamma}_{A, 1} & \boldsymbol{\Gamma}_{B, 1} & \boldsymbol{\Gamma}_{A, 2} & \boldsymbol{\Gamma}_{A, 3} & \boldsymbol{\Gamma}_{A, 4}
\end{array}\right]\left[\begin{array}{c}
f_{1} \\
f_{5} \\
f_{2}+f_{6} \\
f_{3}+f_{7} \\
f_{4}+f_{8}
\end{array}\right]
$$

Consequently, the twist of the link $\mathrm{ABE}$ can be solved with the reciprocal screw theory

$$
\boldsymbol{\Phi}_{A B E}{ }^{T} \mathbf{E} \boldsymbol{\Gamma}_{A B E}=0
$$

Then the twist of the link ABE, denoted by $\boldsymbol{\Phi}_{\mathrm{ABE}}$, is

$$
\boldsymbol{\Phi}_{A B E}=\left\{\begin{array}{c}
0 \\
0 \\
1 \\
\frac{1}{2}\left[y_{A}+y_{B}+\left(x_{B}-x_{A}\right) \cot \alpha\right] \\
\frac{1}{2}\left[\left(y_{A}-y_{B}\right) \tan \alpha-\left(x_{A}+x_{B}\right)\right] \\
0
\end{array}\right\}
$$

The twist of the revolute joint at point $\mathrm{E}$ can be given as

$$
\boldsymbol{\Phi}_{E_{R}}=\left\{\begin{array}{llllll}
0 & 0 & 1 & y_{E} & -x_{E} & 0
\end{array}\right\}^{T}
$$

where $x_{\mathrm{E}}$ is the coordinate of point $\mathrm{E}$ in the $o x$ direction.

Then the twist of joint $\mathrm{E}$ can be given as

$$
\boldsymbol{\Phi}_{E}=\left[\begin{array}{ll}
\boldsymbol{\Phi}_{E_{R}} & \boldsymbol{\Phi}_{A B E}
\end{array}\right]
$$

The terminal constraints exerted to joint $\mathrm{E}$ by the link ABE can be obtained by solving the reciprocal screw equation

$$
\boldsymbol{\Phi}_{E}{ }^{T} \mathbf{E} \boldsymbol{\Gamma}_{E}=0
$$

The terminal constraints are given as 


$$
\boldsymbol{\Gamma}_{E}=\left\{\begin{array}{cccc}
\beta_{1} & 0 & 0 & 0 \\
\beta_{2} & 0 & 0 & 0 \\
0 & 1 & 0 & 0 \\
0 & 0 & 1 & 0 \\
0 & 0 & 0 & 1 \\
\beta_{3} & 0 & 0 & 0
\end{array}\right\}\left[\begin{array}{l}
f_{9} \\
f_{10} \\
f_{11} \\
f_{12}
\end{array}\right]
$$

where

$$
\left\{\begin{array}{c}
\beta_{1}=x_{E}+\frac{1}{2}\left[\left(y_{A}-y_{B}\right) \tan \alpha-\left(x_{A}+x_{B}\right)\right] \\
\beta_{2}=y_{E}-\frac{1}{2}\left[y_{A}+y_{B}-\left(x_{A}-x_{B}\right) \cot \alpha\right] \\
\beta_{3}=-\frac{1}{2}\left\{y_{E}\left[\left(y_{A}-y_{B}\right) \tan \alpha-\left(x_{A}+x_{B}\right)\right]+x_{E}\left[y_{A}+y_{B}-\left(x_{A}-x_{B}\right) \cot \alpha\right]\right\}
\end{array}\right.
$$

Similarly, the terminal constraints exerted to joint E by the link CDE can be obtained as

$$
\boldsymbol{\Gamma}_{E^{\prime}}=\left\{\begin{array}{cccc}
\beta_{1}^{\prime} & 0 & 0 & 0 \\
\beta_{2}^{\prime} & 0 & 0 & 0 \\
0 & 1 & 0 & 0 \\
0 & 0 & 1 & 0 \\
0 & 0 & 0 & 1 \\
\beta_{3}^{\prime} & 0 & 0 & 0
\end{array}\right\}\left[\begin{array}{l}
f_{13} \\
f_{14} \\
f_{15} \\
f_{16}
\end{array}\right]
$$

where

$$
\left\{\begin{array}{c}
\beta_{1}^{\prime}=x_{E}+\frac{1}{2}\left[\left(y_{D}-y_{C}\right) \tan \alpha-\left(x_{D}+x_{C}\right)\right] \\
\beta_{2}^{\prime}=y_{E}-\frac{1}{2}\left[y_{D}+y_{C}-\left(x_{D}-x_{C}\right) \cot \alpha\right] \\
\beta_{3}{ }^{\prime}=-\frac{1}{2}\left\{y_{E}\left[\left(y_{D}-y_{C}\right) \tan \alpha-\left(x_{D}+x_{C}\right)\right]+x_{E}\left[y_{D}+y_{C}-\left(x_{D}-x_{C}\right) \cot \alpha\right]\right\}
\end{array}\right.
$$

It can be found that the GAE will be movable if the terminal constraints exerted to joint $\mathrm{E}$ by the link $\mathrm{ABE}$ and the link $\mathrm{CDE}$ are equal, which is

$$
\boldsymbol{\Gamma}_{E}=\boldsymbol{\Gamma}_{E^{\prime}}
$$

It can be seen from Fig.7 that the relations between the coordinates of the joints and the subtended angle $\alpha$ are 


$$
\left\{\begin{array}{c}
\tan \alpha=\frac{y_{B}}{x_{B}}=\frac{y_{C}}{x_{C}} \\
\tan \alpha=-\frac{y_{A}}{x_{A}}=-\frac{y_{D}}{x_{D}}
\end{array}\right.
$$

\subsection{Type I GAE}

For Type I GAE, the triangle $\triangle \mathrm{ADE}$ and $\triangle \mathrm{BCE}$ are isosceles. For triangle $\triangle \mathrm{ADE}, \mathrm{F}$ is the midpoint of $\mathrm{AD}$, and then line $\mathrm{EF}$ is perpendicular to line $\mathrm{AD}$. Thus its line equation is

$$
y=\cot \alpha x+b
$$

Substituting the coordinates of joint $F$ in Eq. (17) leads to

$$
y=\cot \alpha x-\frac{x_{A}+x_{D}}{\sin 2 \alpha}
$$

For triangle $\triangle \mathrm{BCE}, \mathrm{G}$ is the midpoint of $\mathrm{BC}$, and then line $\mathrm{EG}$ is perpendicular to line BC. Similarly, the equation of line EG can be given as

$$
y=-\cot \alpha x+\frac{x_{B}+x_{C}}{\sin 2 \alpha}
$$

Thus the coordinates of joint E can be obtained by Eqs. (18) and (19) as

$$
\left\{\begin{array}{c}
x_{E}=\frac{x_{A}+x_{B}+x_{C}+x_{D}}{4} \frac{1}{\cos ^{2} \alpha} \\
y_{E}=\frac{x_{B}+x_{C}-x_{A}-x_{D}}{4} \frac{1}{\sin \alpha \cos \alpha}
\end{array}\right.
$$

Substituting Eqs. (16) and (20) into Eq. (12) leads to

$$
\left\{\begin{array}{c}
\beta_{1}=\frac{x_{C}+x_{D}-x_{A}-x_{B}}{4} \sec ^{2} \alpha \\
\beta_{2}=\frac{x_{C}-x_{D}+x_{A}-x_{B}}{2 \sin 2 \alpha} \\
\beta_{3}=\frac{x_{A} x_{C}-x_{B} x_{D}}{2 \sin 2 \alpha \cos ^{2} \alpha}
\end{array}\right.
$$

The coefficients of Eq. (13) can be obtained by substituting Eqs. (16) and (20) into Eq. (14) 


$$
\left\{\begin{array}{c}
\beta_{1}{ }^{\prime}=\frac{x_{A}+x_{B}-x_{C}-x_{D}}{4} \sec ^{2} \alpha \\
\beta_{2}{ }^{\prime}=\frac{x_{B}+x_{D}-x_{A}-x_{C}}{2 \sin 2 \alpha} \\
\beta_{3}{ }^{\prime}=\frac{-x_{A} x_{C}+x_{B} x_{D}}{2 \sin 2 \alpha \cos ^{2} \alpha}
\end{array}\right.
$$

Substituting Eqs. (21) and (22) into Eqs. (11) and (13) respectively, it can be found that the terminal constraints exerted to joint $\mathrm{E}$ by the link $\mathrm{ABE}$ and the link $\mathrm{CDE}$ are equal. That is to say the Type I GAE is movable.

\subsection{Type II GAE}

For Type II GAE, the geometric conditions are

$$
\left\{\begin{array}{c}
\frac{A E}{D E}=\frac{B E}{C E} \\
\angle A E D=\angle B E C
\end{array}\right.
$$

Thus $\triangle \mathrm{ADE}$ and $\triangle \mathrm{BCE}$ are similar triangles which leads to

$$
\frac{A E}{B E}=\frac{D E}{C E}=\frac{A D}{B C}
$$

This can also be rewritten as

$$
\frac{\left(y_{E}-y_{A}\right)^{2}+\left(x_{E}-x_{A}\right)^{2}}{\left(y_{E}-y_{B}\right)^{2}+\left(x_{E}-x_{B}\right)^{2}}=\frac{\left(y_{E}-y_{D}\right)^{2}+\left(x_{E}-x_{D}\right)^{2}}{\left(y_{E}-y_{C}\right)^{2}+\left(x_{E}-x_{C}\right)^{2}}=\frac{\left(y_{A}-y_{D}\right)^{2}+\left(x_{A}-x_{D}\right)^{2}}{\left(y_{B}-y_{C}\right)^{2}+\left(x_{B}-x_{C}\right)^{2}}
$$

Then the coordinates of joint E can be obtained with Eqs. (16) and (25) as

$$
\left\{\begin{array}{c}
x_{E}=\frac{x_{A} x_{C}-x_{B} x_{D}}{x_{A}-x_{B}+x_{C}-x_{D}} \\
y_{E}=\frac{x_{A} x_{C}-x_{B} x_{D}}{x_{A}+x_{B}-x_{C}-x_{D}} \tan \alpha
\end{array}\right.
$$

Substituting Eqs. (16) and (26) into Eq. (12) leads to

$$
\left\{\begin{array}{c}
\beta_{1}=\frac{1}{2} \frac{\left(2 x_{A} x_{C} \cos ^{2} \alpha-2 x_{B} x_{D} \cos ^{2} \alpha+x_{A} x_{D}-x_{A} x_{C}-x_{A}{ }^{2}+x_{B} x_{D}+x_{B}{ }^{2}-x_{B} x_{C}\right)}{\left(x_{A}+x_{C}-x_{B}-x_{D}\right) \cos ^{2} \alpha} \\
\beta_{2}=-\frac{1}{2} \frac{\left(2 x_{A} x_{C} \cos ^{2} \alpha-2 x_{B} x_{D} \cos ^{2} \alpha+x_{A} x_{D}-x_{A} x_{C}-x_{A}{ }^{2}+x_{B} x_{D}+x_{B}{ }^{2}-x_{B} x_{C}\right)}{\left(x_{A}+x_{B}-x_{C}-x_{D}\right) \sin \alpha \cos \alpha} \\
\beta_{3}=-\frac{1}{2} \frac{\left(x_{A} x_{C}-x_{B} x_{D}\right)\left(2 x_{A} x_{C} \cos ^{2} \alpha-2 x_{B} x_{D} \cos ^{2} \alpha+x_{A} x_{D}-x_{A} x_{C}-x_{A}{ }^{2}+x_{B} x_{D}+x_{B}{ }^{2}-x_{B} x_{C}\right)}{\left(x_{A}+x_{B}-x_{C}-x_{D}\right)\left(x_{A}+x_{C}-x_{B}-x_{D}\right) \sin \alpha \cos \alpha}
\end{array}\right.
$$


The coefficients of Eq. (13) can also be obtained by substituting Eqs. (16) and (26) into Eq. (14)

$$
\left\{\begin{array}{c}
\beta_{1}{ }^{\prime}=\frac{1}{2} \frac{\left(2 x_{A} x_{C} \cos ^{2} \alpha-2 x_{B} x_{D} \cos ^{2} \alpha+x_{B} x_{C}-x_{C}{ }^{2}-x_{A} x_{C}+x_{D}{ }^{2}+x_{B} x_{D}-x_{A} x_{D}\right)}{\left(x_{A}+x_{C}-x_{B}-x_{D}\right) \cos ^{2} \alpha} \\
\beta_{2}{ }^{\prime}=-\frac{1}{2} \frac{\left(2 x_{A} x_{C} \cos ^{2} \alpha-2 x_{B} x_{D} \cos ^{2} \alpha+x_{B} x_{C}-x_{C}{ }^{2}-x_{A} x_{C}+x_{D}{ }^{2}+x_{B} x_{D}-x_{A} x_{D}\right)}{\left(x_{A}+x_{B}-x_{C}-x_{D}\right) \sin \alpha \cos \alpha} \\
\beta_{3}{ }^{\prime}=-\frac{1}{2} \frac{\left(x_{A} x_{C}-x_{B} x_{D}\right)\left(2 x_{A} x_{C} \cos ^{2} \alpha-2 x_{B} x_{D} \cos ^{2} \alpha+x_{B} x_{C}-x_{C}{ }^{2}-x_{A} x_{C}+x_{D}{ }^{2}+x_{B} x_{D}-x_{A} x_{D}\right)}{\left(x_{A}+x_{B}-x_{C}-x_{D}\right)\left(x_{A}+x_{C}-x_{B}-x_{D}\right) \sin \cos \alpha}
\end{array}\right.
$$

Combining and rearranging Eqs. (27) and (28) leads to

$$
\begin{aligned}
& \left\{\begin{array}{c}
\frac{\beta_{1}}{\beta_{2}}=-\frac{x_{A}+x_{B}-x_{C}-x_{D}}{x_{A}+x_{C}-x_{B}-x_{D}} \tan \alpha \\
\frac{\beta_{1}}{\beta_{3}}=-\frac{x_{A}+x_{B}-x_{C}-x_{D}}{x_{A} x_{C}-x_{B} x_{D}} \sin \alpha \cos \alpha
\end{array}\right. \\
& \left\{\begin{array}{c}
\frac{\beta_{1}{ }^{\prime}}{\beta_{2}{ }^{\prime}}=-\frac{x_{A}+x_{B}-x_{C}-x_{D}}{x_{A}+x_{C}-x_{B}-x_{D}} \tan \alpha \\
\frac{\beta_{1}{ }^{\prime}}{\beta_{3}{ }^{\prime}}=-\frac{x_{A}+x_{B}-x_{C}-x_{D}}{x_{A} x_{C}-x_{B} x_{D}} \sin \alpha \cos \alpha
\end{array}\right.
\end{aligned}
$$

From Eqs. (29) and (30) it can be shown that

$$
\beta_{1}: \beta_{2}: \beta_{3}=\beta_{1}{ }^{\prime}: \beta_{2}{ }^{\prime}: \beta_{3}{ }^{\prime}
$$

Substituting Eq. (31) into Eqs. (11) and (13), it can be found that the terminal constraints exerted to joint $\mathrm{E}$ by the link $\mathrm{ABE}$ and the link $\mathrm{CDE}$ are equal. That also concludes Type II GAE is movable.

\section{Conclusions}

In this paper, we studied the kinematics of generalized angulated scissor-like elements, 
which forms the planar closed loop linkage. Firstly, every angulated rod was assumed as a PRRP linkage, which has two prismatic joints and two revolute joints. Thus the two PRRP linkages of the GAE are individually movable with a single degree of freedom. The mobility was analyzed with the method based on the screw theory. It has been shown that both types of GAE are movable because the terminal constraints imposed onto the common joint $\mathrm{E}$ by the link $\mathrm{ABE}$ and $\mathrm{CDE}$ are equal. It should be noted that a more general angulated scissor element containing intermediate parallelograms, as reported by You and Pellegrino [14], is yet to be proven with the help of this theory. Further extensions of this approach include the kinematic analysis of planar closed loop double chain linkages which satisfy the loop parallelogram condition [17] or other multi-loop linkages [27].

\section{Acknowledgements}

The work presented in this article was supported by the National Natural Science Foundation of China (Grant No. 51308106, No. 51278116 and No. 51450110080), the Natural Science Foundation of Jiangsu Province (Grant No. BK20130614), the Specialized Research Fund for the Doctoral Program of Higher Education (Grant No. 20130092120018) and a Project Funded by the Priority Academic Program Development of Jiangsu Higher Education Institutions. The first author would like to thank Dr. Zhao Jingshan at Tsinghua University to provide some useful comments and suggestions. Authors also thank the anonymous reviewers for their valuable comments and thoughtful suggestions which improved the quality of the presented work. 


\section{References}

[1] Gantes GJ. Deployable Structures: Analysis of Design. WIT Press, Southampton, Boston, 2001.

[2] Ishii K. Structural Design of Retractable Roof Structures. WIT Press, Southampton, Boston, 2000.

[3] Ingham MD. Microdynamics and Thermal Snap Response of Deployable Space Structures. McGill University, 1995.

[4] Raskin I, Roorda J. Nonlinear Analysis Columns Uniform Pantographic Compression. Journal of Engineering Mechanics, 1999, 12: 1344-1348.

[5] Langbecker, T. Kinematic analysis of deployable scissor structures. International Journal of Space Structures, 1999, 14 (1):1-15.

[6] Rosenfeld Y, Logcher RD. New concepts for deployable collapsible structures. International Journal of Space Structures, 1988, 3 (1):20-32.

[7] Escrig F, Valcarcel JP, Sanchez J. Deployable cover on a swimming pool in Seville. Journal of the International Association for Shell and Spatial Structures, 1996, 37 (1): 39-70.

[8] Gantes CJ, Konitopoulou E. Geometric design of arbitrarily curved bi-stable deployable arches with discrete joint size. International Journal of Solids and Structures, 2004, 41: 5517-5540.

[9] Van Mele T. Scissor-Hinged Retractable Membrane Roofs, Vrije Universiteit Brussel, 2008.

[10] Zhao JS, Wang JY, Chu FL, Feng ZJ, Dai JS. Mechanism Synthesis of a foldable stair. Journal of Mechanism and Robotics, 2012, 4: 014502. 
[11] Zhao JS, Wang JY, Chu FL, Feng ZJ, Dai JS. Structure synthesis and static analysis of a foldable stair. Mechanism and Machine Theory, 2011, 46:998-1015.

[12] Hoberman C. Reversibly expandable doubly-curved truss structures. US Patent 4,942,700

[13] Hoberman C. Radial expansion/retraction truss structures. US Patent 5,024,031

[14] You Z, Pellegrino S. Foldable Bar Structures. International Journal of Solids and Structures. 1997, 34(15): 1825-1847.

[15] You Z. Deployable Structure of Curved Profile for Space Antennas. Journal of Aerospace Engineering ASCE, 2000, 13(4): 139-143.

[16] Patel J, Ananthasuresh GK. A kinematic theory for radially foldable planar linkages. International Journal of Solids and Structures. 2007, 44: 6279-6298.

[17] Mao DC, Luo YZ, You Z. Planar Closed Loop Double Chain Linkages. Mechanism and Machine Theory, 2009, 44(4): 850-859.

[18] Nagaraj BP, Pandiyan R, Ghosal A. Kinematics of pantograph masts. Mechanism and Machine Theory, 2009, 44(4):822-834.

[19] Dai J S, Rees Jone J. Mobility in metamorphic mechanisms of foldable/erectable kinds. Journal of Mechanical Design, Transactions of ASME, 1999, 121(3):375-382.

[20] Dai, J.S. and Rees Jones, J., Null-space construction using cofactors from a screw algebra context, Proceedings of the Royal Society of London. Series A: Mathematical, Physical and Engineering Sciences, 458 (2024): 1845-1866, 2002.

[21] Dai, J.S., Huang, Z., Lipkin, H., Mobility of Overconstrained Parallel Mechanisms, Special 
Supplement on Spatial Mechanisms and Robot Manipulators, Transactions of the ASME: Journal of Mechanical Design, 128(1): 220-229, 2006.

[22] Zhao JS, Chu FL, Feng ZJ. The mechanism theory and application of deployable structures based on SLE. Mechanism and Machine Theory, 2009, 44(2): 324-335.

[23] Cai JG, Xu YX, Feng J. Kinematic analysis of Hoberman's Linkages with the screw theory. Mechanism and Machine Theory, 2013, 63: 28-34.

[24] Dai J S, Li D, Zhang Q, Jin G G. Mobility analysis of a complex structured ball based on mechanism decomposition and equivalent screw system analysis. Mechanism and Machine Theory, 2004, 39: 445-458.

[25] Wei, G., Ding, X. and Dai, J.S., Mobility and geometric analysis of the Hoberman switch-pitch ball and its variant, Journal of Mechanisms and Robotics, Trans. ASME, 2(3): 031010, 2010.

[26] Wei, G. and Dai, J.S., A spatial eight-bar linkage and its association with the deployable platonic mechanisms, Journal of Mechanisms and Robotics, Trans. ASME, 6(5), 2014.

[27] Baker JE, On Mobility and Relative Freedoms in Multiloop Linkages and Structures, Mechanism and Machine Theory, 1981, 16(6): 583-597. 


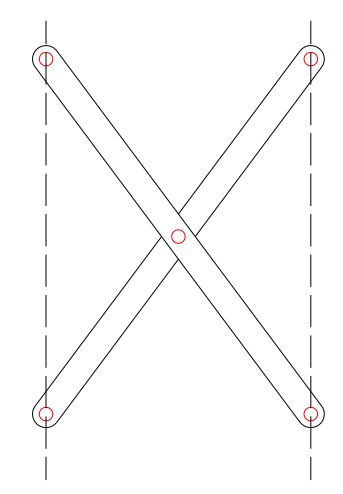

(a)

(b)

Fig.1 Scissor-like elements.

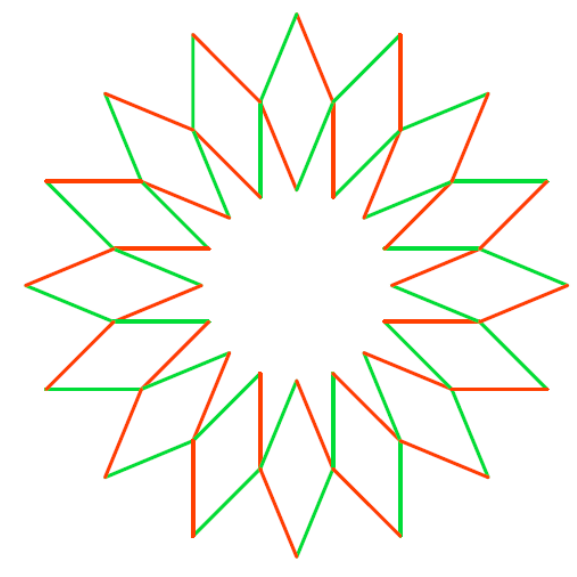

(a)

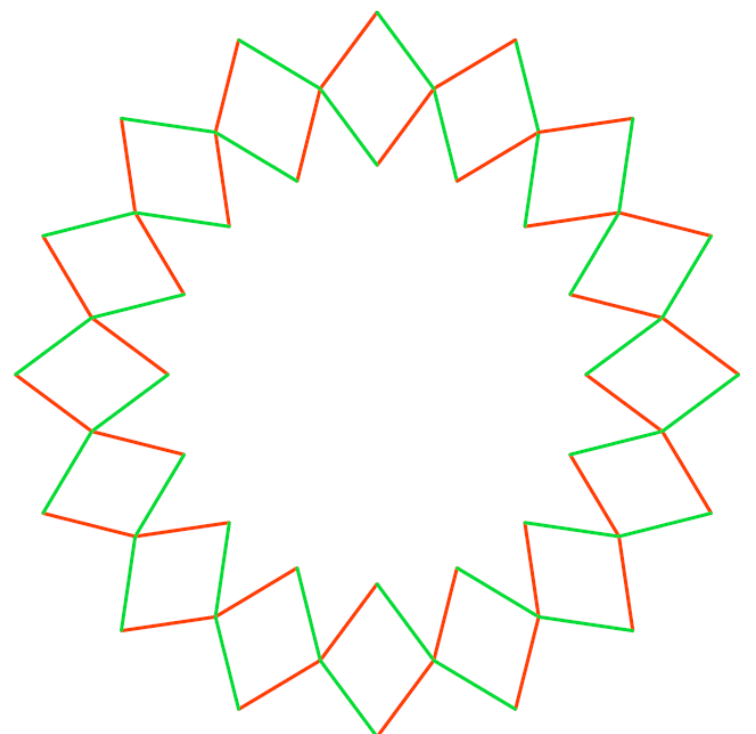

(b)

Fig.2 Hoberman's Linkage. 

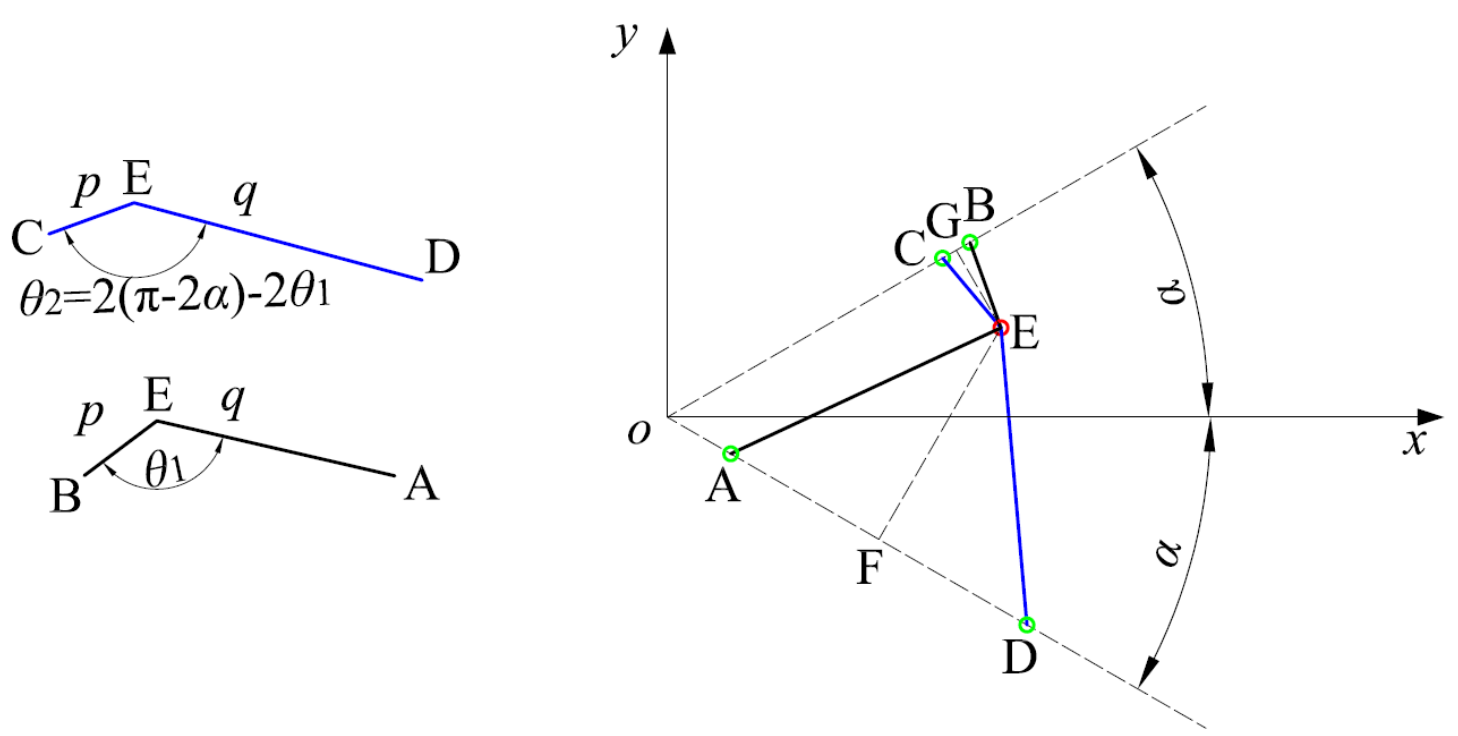

Fig.3 Type I GAE formed by angulated rods
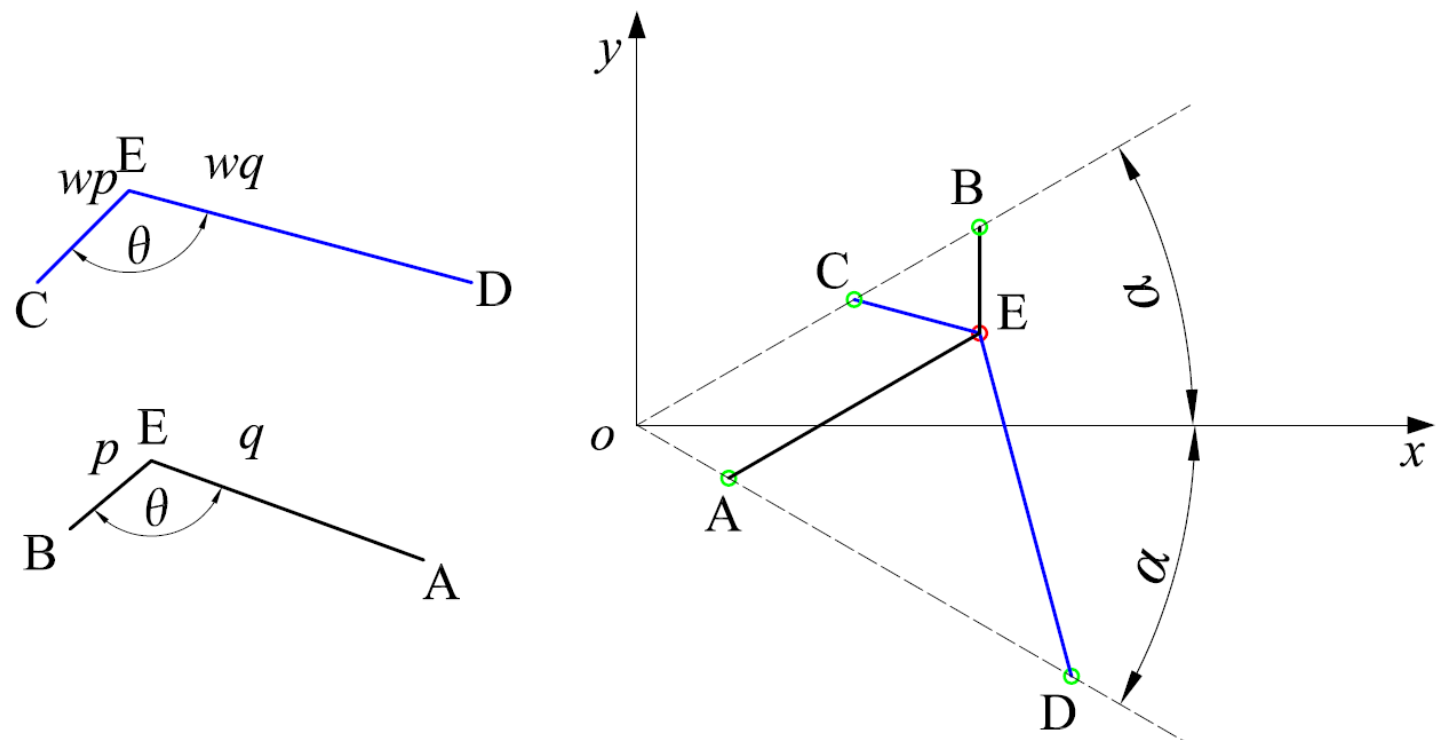

Fig.4 Type II GAE formed by angulated rods 


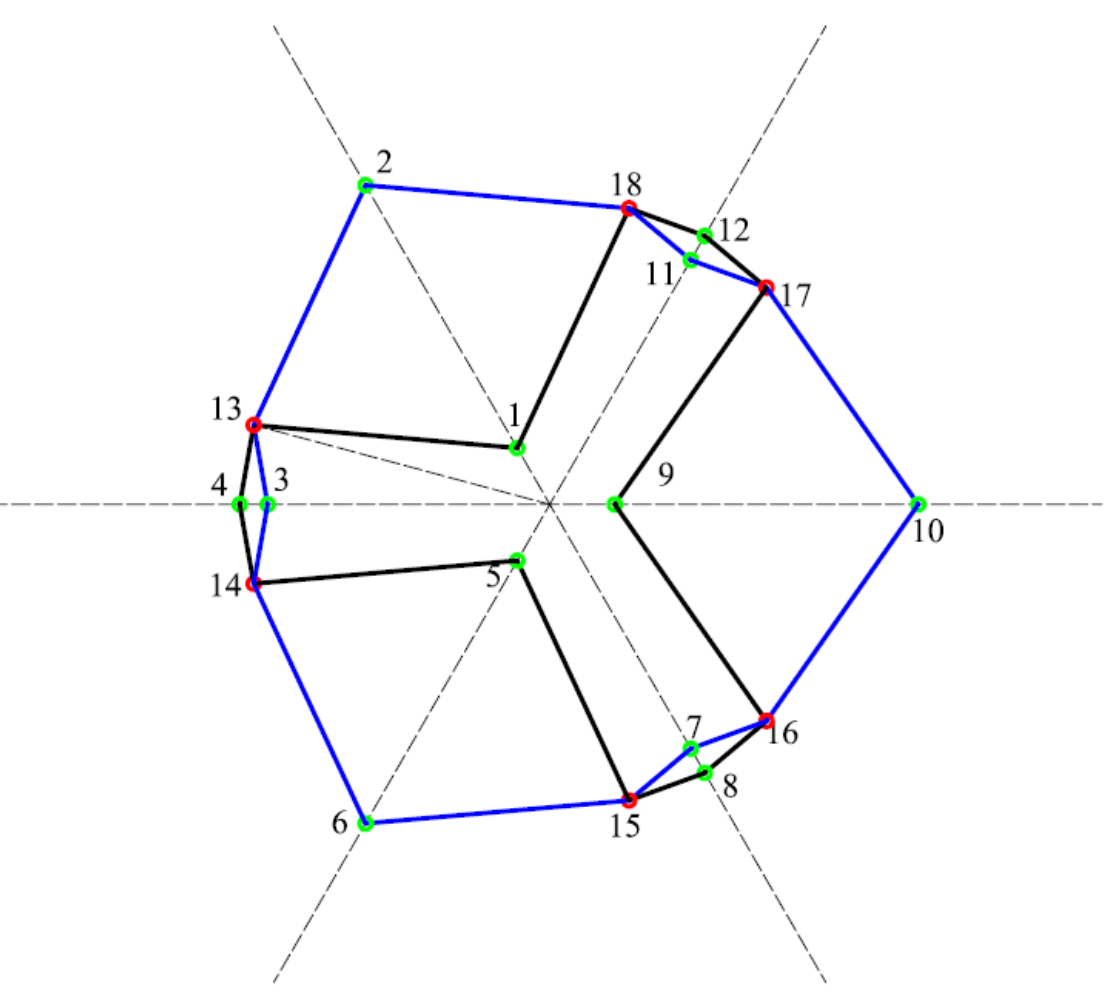

(a) Type I

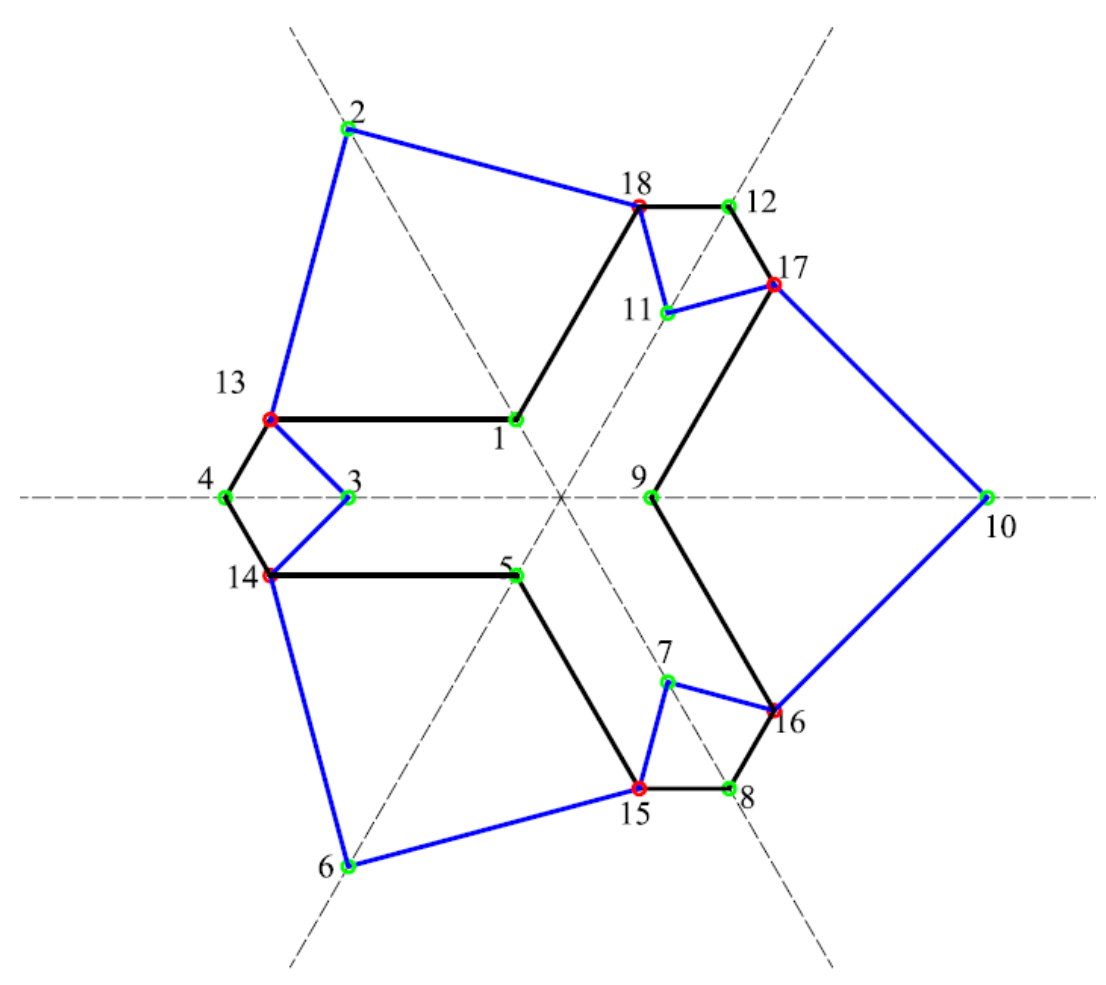

(b) Type II

Fig. 5 Planar closed-loop linkages with GAEs 


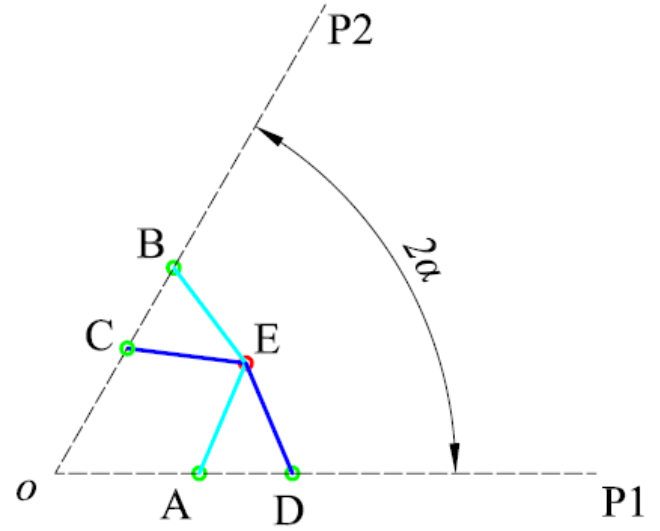

(a)

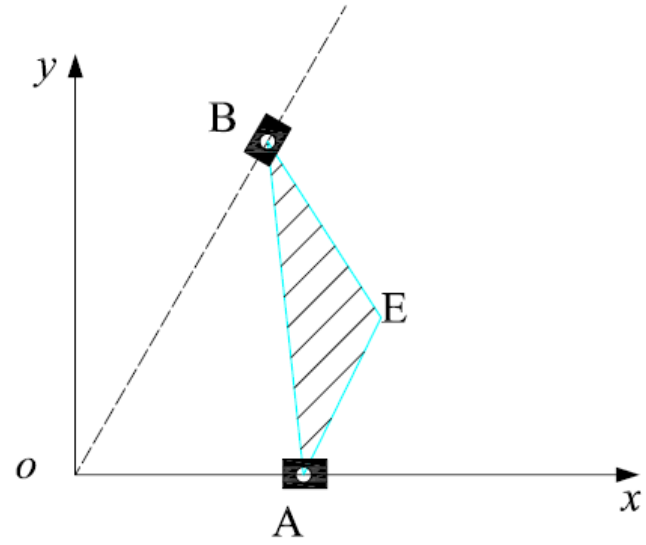

(b)

Fig.6 Kinematic description of GAEs.

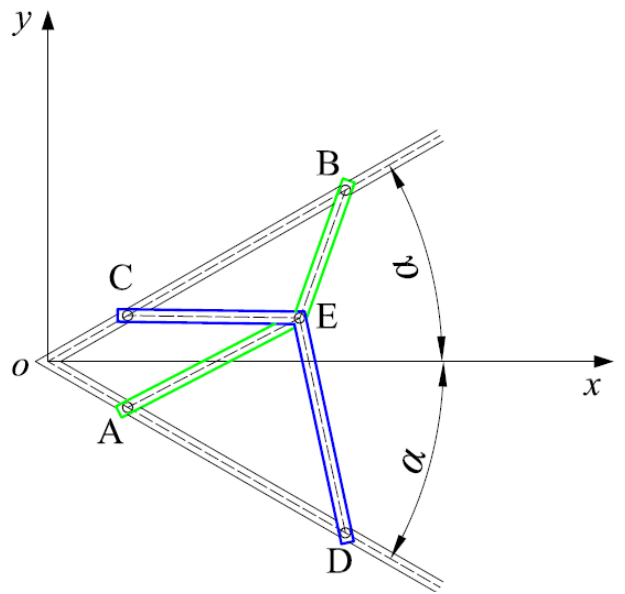

Fig.7 Geometric description of GAEs. 